\title{
Severe Weight Loss and Dropped Head Syndrome in MuSK Myasthenia Gravis
}

\author{
Daniel Yu Bing Tan1, Willem Hendrik Joseph Peter Linssen", Boris Liberov² \\ ${ }^{1}$ Department of Neurology, Sint Lucas Andreas Hospital, Amsterdam, The Netherlands \\ ${ }^{2}$ Department of Internal Medicine, Zaans Medisch Centrum, The Netherlands \\ Email: d.tan@slaz.nl
}

Received 21 September 2014; revised 15 October 2014; accepted 6 November 2014

Copyright (C) 2014 by authors and Scientific Research Publishing Inc.

This work is licensed under the Creative Commons Attribution International License (CC BY).

http://creativecommons.org/licenses/by/4.0/

c) (i) Open Access

\begin{abstract}
Muscle Specific Receptor Thyrosine Kinase (MuSK) Myasthenia Gravis (MG) is a rare auto-immune disorder of the neuromuscular junction. The clinical presentation of MG is dominated by fluctuating weakness of the extra-ocular, orofacial and limb muscles. The clinical presentation of MuSK MG can vary, which may delay diagnostic procedures. We present a patient who initially presented with severe weight loss and slowly progressive developing neck extensor weakness and diplopia. A single fiber EMG led to the diagnosis MG and antibodies directed towards MuSK were detected. He was treated with prednisone and the steroid sparing agent azathioprine after which he made full recovery.
\end{abstract}

\section{Keywords}

Myasthenia Gravis, Muscle Specific Receptor Thyrosine Kinase, Dropped Head Syndrome

\section{Introduction}

Acquired myasthenia gravis (MG) is a well-known neuromuscular junction disorder. The clinical picture is characterized by fluctuating muscle weakness that increases with effort. In most cases MG starts with eye symptoms, gradually extending to generalized fluctuating muscle weakness. Patients may complain about ptosis, diplopia, dysarthria, dysphagia, dyspnoea, facial weakness or fatigable limb or axial weakness. After clinical evaluation, the diagnosis is usually confirmed by electrodiagnostic studies, either by repetitive nerve stimulation (RNS) or by single fiber electromyography (SFEMG) [1]. MG is an antibody mediated autoimmune disease. Currently several relevant antibodies have been identified that play a role in the pathophysiology of MG. These include antibodies directed towards the skeletal muscle acetylcholine receptor (AChR) [2] and antibodies directed towards MuSK [3], (Figure 1). In 85\% of patients diagnosed with generalized MG, antibodies to AChR 


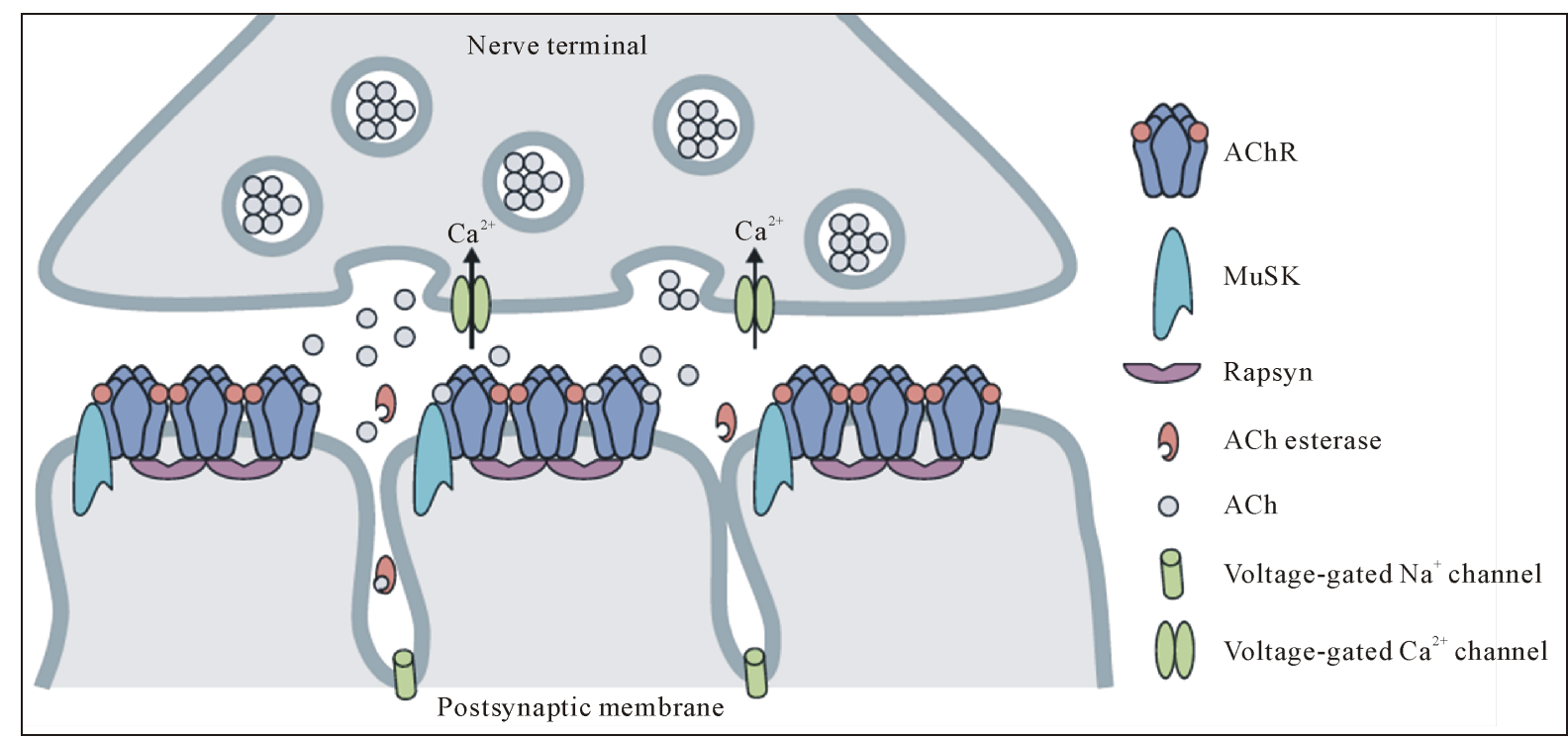

Figure 1. Components of the neuromuscular junction. The AChR is localized on the post-synaptic membrane and receives ACh following a nerve action potential that releases Ach from presynaptic vesicles. MuSK is considered important in maintaining a normal post synaptic membrane functioning. ACh = acetylcholine, AChR = ACh receptor, MuSK = musclespecific receptor thyrosine kinase. Reprinted with permission from Elsevier [16].

are detected using sensitive radioimmunoprecipitation assays. In case of anti-AChR seronegativity, $40 \%$ of patients will have antibodies to MuSK [4]. More recently antibodies towards low-density lipoprotein receptor-related protein 4 (Lrp4) and Agrin have been identified [5] [6]. These different antibody profiles have distinct clinical features and should be recognized as they are relevant for prognosis and treatment [7].

We present a patient with severe weight loss and, initially subtle but later progressive, neurological deficits including neck extensor weakness and diplopia. He was confronted with considerable doctors delay due to an evaluation by medical specialists with different expertise, but unfamiliar with the clinical features of MuSK MG.

\section{Clinical Record}

A 41-year-old male presented to the neurology outpatient clinic after having visited various other medical specialists in the past half year. His complaints started about 6 months earlier with double vision. The diplopia was constantly present and did not fluctuate during the day. The ophthalmologist noticed a limitation in the adduction of both eyes and prescribed prism-glasses. These glasses gave a short lived relief of symptoms. In the weeks following the diplopia, the patient noticed difficulties in swallowing. Mainly firm foods, not liquid substances, were a problem. He reported the food was hard to process due to fatigability of chewing muscles. The patient lost $10 \mathrm{~kg}$ of weight within a period of 8 weeks. Visits to the ENT doctor and the internal medicine, where laryngoscopies, gastroscopies and a video of the swallow-reflex where performed, did not result in a satisfactory explanation.

About three months after the diplopia and dysphagia had started, the clinical picture progressed and the patient noticed difficulty in keeping his head up straight. He developed a severe dropped head syndrome and was only capable of looking straight forward by manually lifting his head. He was now referred to the neurology outpatient clinic for further examination. History taking reported no headaches, no nausea, no changes in vision or hearing, no muscle weakness or numbness of the extremities and no inflammatory symptoms in the last few months. He had a blank medical history and used no medication, drugs or alcohol. He had a catering industry job and worked out in the gym. His family history mentioned no neuromuscular diseases. On neurological examination we saw a cachectic man, without apparent localized muscle atrophy and no generalised muscle weakness. He had severe weakness of the neck extensor muscles leading to a dropped head syndrome, forcing him to lift his head with both hands. This manoeuvre enabled him to keep his head upright for a few seconds after which his head fell forward again. He had definite limitations in vertical and horizontal eye movements and only mild weakness of the facial musculature. He had normal strength, tendon reflexes and sensibility in the up- 
per and lower extremities and these muscles did not appear fatigable by exercise.

The patient was admitted to the neurology ward for investigation of a possible neuromuscular junction disorder. Extensive blood tests were performed showing normal haematology, kidney, liver and thyroid functions, electrolytes and vitamin status. Serologic tests for Borrelia burgdorferi and Treponema pallidum were negative. A MRI scan of the brain and the cervical spine were performed and showed no structural abnormalities. Because a disorder of the neuromuscular junction was suspected extensive electrodiagnostic studies were performed. Nerve conduction studies, both sensory and motor, were normal. Repetitive nerve stimulation of the muscles at the extremities showed some decrement of the compound muscle action potentials (CMAP), not followed by an increment after rest. A neostigmine-test for diagnosing AChR MG was done and was found negative for all symptoms. Eventually a single fibre EMG of the orbicularis oculi muscle was performed which showed an extended jitter with frequent blocking of excitation at the neuromuscular junction, confirming neuromuscular junction disease. Antibodies against MuSK were determined and showed positive. Pathology of the thymus was excluded after obtaining a CT scan of the thorax.

The patient was treated with high doses of prednisone in combination with the steroid sparing agent azathioprine. He made a rapid recovery. A year after treatment all muscle complaints had subsided and the patient was fully back to work, he even considered going back to the gym.

\section{Discussion}

This case illustrates doctor's delay in a patient with a rare neuromuscular junction disorder and slowly progressive neurological symptoms. Different medical specialists excluded pathology in their own field of expertise but were unfamiliar with the disease. On neurological evaluation MG was diagnosed, confirmed by the detection of antibodies directed towards MuSK.

MG is a relatively uncommon disease [8]. In the Netherlands the prevalence of generalised MG subtypes per one million inhabitants is 1.9 (CI: 1.22 - 2.59) for MuSK MG, 90.0 (CI: 75.8 - 104.2) for AChR MG and 10.1 (CI: 5.3 - 14.9) for seronegative MG. The incidence rates per million person years are 0.10 (CI: $0.06-0.14$ ), 4.64 (CI: 3.76 - 5.52) and 0.38 (CI: 0.13 - 0.63) respectively [9].

Clinically patients with myasthenic syndromes usually present with fatigability and fluctuating muscle weakness. In $85 \%$ of patients with generalized (most AChR) MG the initial symptoms are ocular, with either ptosis, diplopia or ptosis and diplopia [10].

Our patient presented with diplopia, dysphagia and a dropped head syndrome. In MuSK MG, the presenting symptoms may be ptosis and or diplopia as in AChR positive patients, but MuSK positive patients characteristically show progressive weakness of the bulbar muscles leading to dysarthria and swallowing difficulty. The bulbar muscles usually remain most severely affected throughout the course of the disease [7] [11]. Neck extensor weakness is seen in AChR MG also, but appears to be more common in patients with antibodies to MuSK, occurring in $20 \%-70 \%$ of patients [12] [13]. Finally, respiratory crises are common in MuSK MG and the involvement of limb muscles weakness and fatigability is less severe and more inconsistent compared with AChR MG.

Diagnostic studies in a patient with suspected MG are aimed at objectifying electrodiagnostic abnormalities, either with RNS or by SFEMG. It is electrophysiologically impossible to distinguish between MuSK positive patients and MuSK negative patients. Due to the distribution of the most prevalent clinical characteristics of the different MG subtypes there are however differences in the sensitivity of these tests when different muscles are tested. As ocular, neck, shoulder and bulbar muscles are predominantly affected in MuSK positive patients, RNS and SFEMG of the distal extremities are often normal, while testing of the neck, shoulder and ocular muscles is abnormal [14] [15].

In Figure 1 a schematic drawing of the neuromuscular junction is presented. In this figure it is made clear that ACh is released from the nerve terminal following a nerve action potential. This process is facilitated by the influx of $\mathrm{Ca}^{2+}$ through voltage-gated $\mathrm{Ca}^{2+}$ channels. ACh then interacts with the AChR on the postsynaptic membrane, causing a local depolarisation; the endplate potential (EPP). Acetylcholinesterase terminates the action of ACh on the post synaptic membrane. In AChR MG, AChR's are lost and a sufficient EPP for muscle fibre action potential cannot be reached resulting in neuromuscular transmission failure. MuSK is thought to initiate the clustering of AChR's on the postsynaptic membrane, thus maintaining a normal postsynaptic architecture. This process is adversely affected in MuSK MG leading to reduced numbers of functional AChR's at the site of the 
synapse [16].

The treatment of MG consists of symptomatic therapy plus short- and/or long-term immune therapies. Symptomatic therapy usually consists of the cholinesterase inhibitor pyridostigmine (oral dose, 15 - $60 \mathrm{mg}$ every 4 - 6 hours). Differences in response rates in the various subtypes of MG to cholinesterase inhibitors have been reported in several studies. Non responsiveness to cholinesterase inhibitors seems to be much more common in MuSK MG when compared to seronegative or AChR MG [17] [18]. If it is desirable to achieve a rapid clinical response, for instance in patients with a respiratory crisis or during severe exacerbations, short term immune therapies such as plasma exchange and intravenous immunoglobulin should be considered [19] [20]. Long-term immunosuppressive agents usually consist of corticosteroids alone or in combination with steroid sparing agents such as azathioprine. Finally the thymus is known to play an important role in the pathogenesis of AChR MG. In this subgroup thymectomy can lead to significant improvement of symptoms and should be considered in its management [21]. Thymectomy in the management of anti-MuSK positive MG patients, however, is less clear [22].

\section{Conclusion}

This case illustrates that knowledge about signs and symptoms of MG and specifically of anti-MuSK MG may prevent doctors delay, enabling to start beneficial treatment as soon as possible for these patients.

\section{Acknowledgements}

We are indebted to Prof. Dr. J.J.G.M. Verschuuren for his advice and reading of the manuscript.

\section{References}

[1] Benatar, M. (2006) A Systematic Review of Diagnostic Studies in Myasthenia Gravis. Neuromuscular Disorders, 16, 459-467. http://dx.doi.org/10.1016/j.nmd.2006.05.006

[2] Patrick, J. and Lindstrom, J. (1973) Autoimmune Response to Acetylcholine Receptors. Science, 180, 871-872. http://dx.doi.org/10.1126/science.180.4088.871

[3] Hoch, W., McConville, J., Helms, S., Newsome-Davis, J., Melms, A. and Vincent, A. (2001) Auto-Antibodies to the Receptor Tyrosine Kinase MuSK in Patients with Myasthenia Gravis without Acetylcholine Receptor Antibodies. Nature Medicine, 7, 365-368. http://dx.doi.org/10.1038/85520

[4] McConville, J., Farrugia, M.E., Beeson, D., Kishore, U., Metcalfe, R., Newsome-Davis, J. and Vincent, A. (2004) Detection and Characterisation of MuSK Antibodies in Seronegative Myasthenia Gravis. Annals of Neurology, 55, 580584. http://dx.doi.org/10.1002/ana.20061

[5] Pevzner, A., Schoser, B., Peters, K., Cosma, N., Karakatsani, A., Schalke, B., et al. (2012) Anti-LRP4 Autoantibodies in AChR- and MuSK-Antibody-Negative Myasthenia Gravis. Journal of Neurology, 259, 427-435. http://dx.doi.org/10.1007/s00415-011-6194-7

[6] Gasperi, C., Melms, A., Schoser, B., Zhang, Y., Meltoranta, J., Risson, V., et al. (2014) Anti-Agrin Autoantibodies in Myasthenia Gravis. Neurology, 82, 1976-1983. http://dx.doi.org/10.1212/WNL.0000000000000478

[7] Deymeer, F., Gungor-Tuncer, O., Yilmaz, V., Parman, Y., Serdaroglu, P., Ozdemir, C., Vincent, A. and Saruhan-Direskeneli, G. (2007) Clinical Comparison of Anti-MuSK- vs Anti AChR-Positive and Seronegative Myasthenia Gravis. Neurology, 68, 609-611. http://dx.doi.org/10.1212/01.wnl.0000254620.45529.97

[8] Wirtz, P.W., Nijnuis, M.G., Sotodeh, M., Willems, L.N.A., Brahim, J.J., Putter, H., Wintzen, A.R. and Verschuuren, J.J. (2003) The Epidemiology of Myasthenia Gravis, Lambert-Eaton Myasthenic Syndrome and Their Associated Tumours in the Northern Part of the Province of South Holland. Journal of Neurology, 250, 698-701. http://dx.doi.org/10.1007/s00415-003-1063-7

[9] Niks, E.H., Kuks, J.B.M. and Verschuuren, J.J.G.M. (2007) Epidemiology of Myasthenia Gravis with Anti-Muscle Specific Kinase Antibodies in the Netherlands. Journal of Neurology, Neurosurgery and Psychiatry, 78, 417-418. http://dx.doi.org/10.1136/jnnp.2006.102517

[10] Grob, D., Brunner, N., Namba, T. and Pagala, M. (2008) Lifetime Course of Myasthenia Gravis. Muscle Nerve, 37, 141-149. http://dx.doi.org/10.1002/mus.20950

[11] Evoli, A., Tonali, P.A., Padua, L., Lo Monaco, M., Scuderi, F., Batocchi, A.P., Marino, M. and Bartoccioni, E. (2003) Clinical Correlates with Anti-MuSK Antibodies in Generalized Seronegative Myasthenia Gravis. Brain, 126, 23042311. http://dx.doi.org/10.1093/brain/awg223 
[12] Stickler, D.E., Massey, J.M. and Sanders, D.B. (2005) MuSK-Antibody Positive Myasthenia Gravis: Clinical and Electrodiagnostic Patterns. Clinical Neurophysiology, 116, 2065-2068. http://dx.doi.org/10.1016/j.clinph.2005.06.003

[13] Zhou, L., McConville, J., Chaudhry, V., Adams, R.N., Skolasky, R.L., Vincent, A. and Drachman, D.B. (2004) Clinical Comparison of Muscle-Specific Tyrosine Kinase (MuSK) Antibody-Positive and -Negative Myasthenic Patients. Muscle \& Nerve, 30, 55-60. http://dx.doi.org/10.1002/mus.20069

[14] Sanders, D.B., El-Salem, K., Massey, J.M., McConville, J. and Vincent, A. (2003) Clinical Aspects of MuSK Antibody Positive Seronegative MG. Neurology, 60, 1978-1980. http://dx.doi.org/10.1212/01.WNL.0000065882.63904.53

[15] Pasnoor, M., Wolfe, G.I., Nations, S., Trivedi, J., Barohn, R.J., Herbelin, L., et al. (2010) Clinical Findings in MuSK-Antobody Positive Myasthenia Gravis: A U.S. Experience. Muscle \& Nerve, 41, 370-374. http://dx.doi.org/10.1002/mus.21533

[16] Meriggioli, M.N. and Sanders, D.B. (2009) Autoimmune Myasthenia Gravis: Emerging Clinical and Biological Heterogeneity. Lancet Neurology, 8, 475-490. http://dx.doi.org/10.1016/S1474-4422(09)70063-8

[17] Hatanaka, Y., Hemmi, S., Morgan, M.B., Scheufele, M.L., Claussen, G.C., Wolfe, G.I. and Oh, S.J. (2005) Nonresponsiveness to Anticholinesterase Agents in Patients with MuSK-Antibody-Positive MG. Neurology, 65, 1508-1509. http://dx.doi.org/10.1212/01.wnl.0000183145.91579.74

[18] Evoli, A. and Padua, L. (2013) Diagnosis and Therapy of Myasthenia Gravis with Antibodies to Muscle-Specific Kinase. Autoimmunity Reviews, 12, 931-935. http://dx.doi.org/10.1016/j.autrev.2013.03.004

[19] Gajdos, P., Chevret, S., Clair, B., Tranchant, C. and Chastang, C. (1997) Clinical Trial of Plasma Exchange and High Dose Intravenous Immunoglobulin in Myasthenia Gravis. Annals of Neurology, 41, 789-796. http://dx.doi.org/10.1002/ana.410410615

[20] Qureshi, A.L., Chaundry, M.A., Akbar, M.S., Mohammad, Y., Chua, H.C., Yahia, A.M., et al. (1999) Plasma Exchange versus Intravenous Immunoglobulin Treatment in Myasthenic Crisis. Neurology, 52, 629-632. http://dx.doi.org/10.1212/WNL.52.3.629

[21] Gronseth, G.S. and Barohn, R.J. (2000) Practice Parameter: Thymectomy for Autoimmune Myasthenia Gravis (an Evidence-Based Review): Report of the Quality Standards Subcommittee of the American Academy of Neurology. Neurology, 55, 7-15. http://dx.doi.org/10.1212/WNL.55.1.7

[22] Leite, M.I., Ströbel, P., Jones, M., Micklem, K., Moritz, R., Gold, R., et al. (2005) Fewer Thymic Changes in MuSK Antibody-Positive than in MuSK Antibody-Negative MG. Annals of Neurology, 57, 444-448. http://dx.doi.org/10.1002/ana.20386 
Scientific Research Publishing (SCIRP) is one of the largest Open Access journal publishers. It is currently publishing more than 200 open access, online, peer-reviewed journals covering a wide range of academic disciplines. SCIRP serves the worldwide academic communities and contributes to the progress and application of science with its publication.

Other selected journals from SCIRP are listed as below. Submit your manuscript to us via either submit@scirp.org or Online Submission Portal.
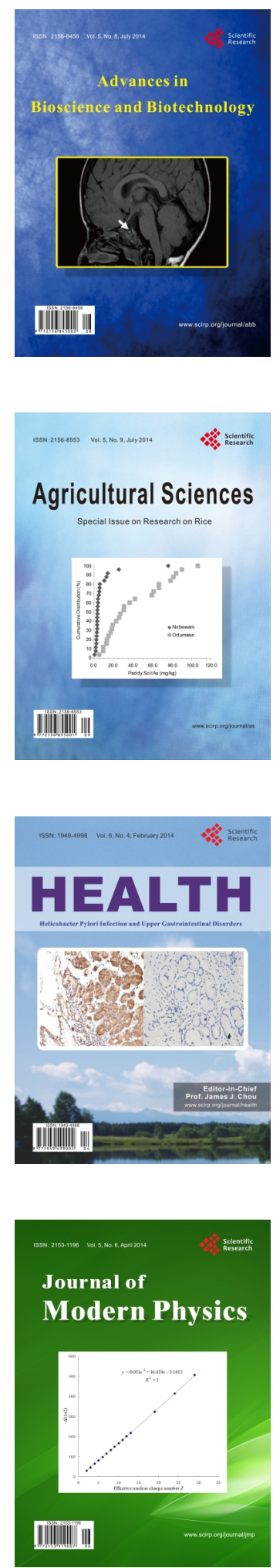
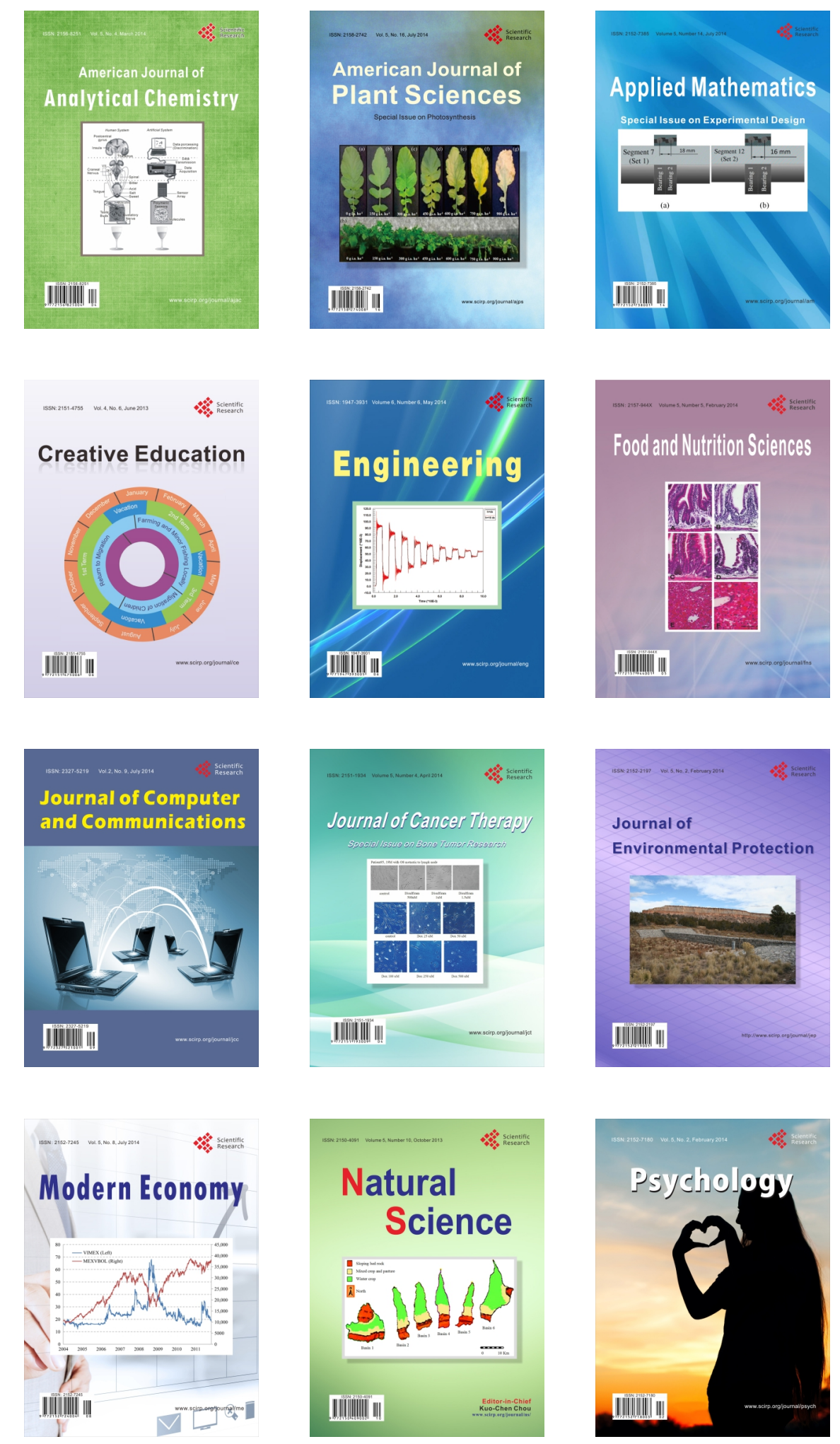